\title{
Tramadol/Acetaminophen Combination Tablets in Cancer Patients with Chemotherapy-Induced Peripheral Neuropathy: A Single-Arm Phase II Study
}

Daisuke Naruge, MD, PhD, ${ }^{*}$ Fumio Nagashima, MD, PhD, Kirio Kawai, MD, DDS, PhD, Naohiro Okano, MD, PhD, Takaaki Kobayashi, MD, PhD, and Junji Furuse, MD, PhD

\begin{abstract}
Background: Chemotherapy-induced peripheral neuropathy (CIPN) is a frequent complication in patients receiving anticancer chemotherapy, but no effective treatment is yet available.

Objective: To evaluate the efficacy and safety of a tramadol/acetaminophen combination tablets for CIPN.

Design: This is a single-arm phase II study of tramadol/acetaminophen.

Setting/subjects: Eligible patients had received oxaliplatin, paclitaxel, or nab-paclitaxel, and were experiencing CIPN. The patients were given one tablet (37.5 mg tramadol plus $325 \mathrm{mg}$ acetaminophen) twice a day for 7 days, then four times a day for 21 days.

Measurements: The primary endpoint was the numerical rating scale of neuropathic pain. Other endpoints included the potential of CYP2D6 genetic variants to effective response or toxicity.

Results: Of the 34 patients enrolled, 23 completed the protocol treatment. The mean neuropathic pain score decreased insignificantly from 5.53 at baseline to 5.00 at 28 days (95\% confidence interval -0.21 to 1.43; $p=0.139$ ). However, 13 of the 23 (56.5\%) patients who completed the protocol treatment showed improvement of the neuropathic pain score by at least 1 point. No severe adverse events were observed. Tramadol/acetaminophen may be more effective in patients with the intermediate metabolizer phenotype of the CYP2D6 single nucleotide polymorphisms (SNPs) although at the cost of increased toxicity.

Conclusions: Although tramadol/acetaminophen tablets did not reduce neuropathic pain to a statistically significant degree, the neuropathic pain severity reduced in more than a half of the patients.
\end{abstract}

Keywords: acetaminophen; anticancer chemotherapy; chemotherapy-induced peripheral neuropathy; CYP2D6; tramadol

\section{Introduction}

Supportive care for chemotherapy-related adverse effects is vitally important to improving the survival and quality of life of cancer patients. Several anticancer drugs frequently cause chemotherapy-induced peripheral neuropathy (CIPN). ${ }^{1}$ Oxaliplatin (L-OHP), ${ }^{2}$ paclitaxel (PTX), and nanoparticle albumin-bound paclitaxel $(\text { nab-PTX })^{3}$ are important agents in the treatment of unresectable gastrointestinal cancers but are severely neurotoxic. Their administration is usually continued for more than six months, and in many cases, the symptom of CIPN lasts after graduating anticancer chemotherapy. CIPN not only impacts upon patient quality of life, but also affects the efficacy of cancer chemotherapy, as dose-limiting toxicity may necessitate chemotherapy reduction or discontinuation. Although

Department of Medical Oncology, Kyorin University Faculty of Medicine, Tokyo, Japan.

*Address correspondence to: Daisuke Naruge, MD, PhD, Department of Medical Oncology, Kyorin University Faculty of Medicine, Shinkawa 6-20-2, Mitaka-shi, Tokyo 181-8611, Japan, E-mail: syuyo-naika@ks.kyorin-u.ac.jp

(c) Daisuke Naruge et al., 2020; Published by Mary Ann Liebert, Inc. This Open Access article is distributed under the terms of the Creative Commons License (http://creativecommons.org/licenses/by/4.0), which permits unrestricted use, distribution, and reproduction in any medium, provided the original work is properly cited. 
many agents have been examined for their efficacy against CIPN in clinical trials, only the serotoninnoradrenaline reuptake inhibitor (SNRI) duloxetine has to date been demonstrated to show some efficacy against CIPN in a phase III study. ${ }^{4}$ The efficacy of duloxetine is not clinically robust, however, and the development of more effective treatments for CIPN is becoming more urgent.

Tramadol hydrochloride not only serves as a $\mu$-opioid receptor agonist, but also acts as an SNRI, activating the descending pain-inhibitory pathways. ${ }^{5}$ Acetaminophen is metabolized to AM404, which also activates the descending pain-inhibitory pathways through the cannabinoid receptors. ${ }^{6}$ Tramadol/acetaminophen combination is, therefore, held to exert a synergistic effect against neuropathic pain, and is generally used for the treatment of neuralgias (e.g., spinal canal stenosis and diabetic neuropathy). ${ }^{7}$ Although the tramadol/ acetaminophen combination tablet (TACT) may be expected to reduce the severity of the symptoms in CIPN, only one clinical trial of TACT against CIPN limiting to L-OHP has been reported to date. ${ }^{8}$

Cytochrome P450 2D6 (CYP2D6) metabolizes tramadol to O-desmethyltramadol (M1). Many SNPs of CYP2D6 have been reported. ${ }^{9,10}$ Although CYP2D6 genetic variants have the potential to become useful biomarkers of the efficacy and/or toxicity of tramadol, no clinical study for CIPN have been conducted. And CYP2D6 does not affect the metabolism of acetaminophen.

Hypothesizing that the TACT would be effective for reducing the neuropathic pain of CIPN, we conducted this phase II study to evaluate its efficacy and safety against CIPN. We also investigated genetic variations in CYP2D6 as a predictive marker of the efficacy and toxicity of TACT.

\section{Patients and Methods \\ Patients}

This study was conducted in a single center, Kyorin University Hospital. Eligible patients were those who had previously received or were currently receiving anticancer chemotherapy, which included L-OHP, PTX, or nab-PTX, and had at least grade 2 peripheral sensory neuropathy, diagnosed based on the National Cancer Institute Common Terminology Criteria for Adverse Events version 4.0 (CTCAE v4.0), persisting for more than or equal totwo weeks. Other eligibility criteria included age $\geq 20$ years, an Eastern Cooperative Oncology Group performance status of 0 to 2, and capability of oral intake for at least 4 weeks. Concomitant use of nonsteroidal anti-inflammatory drugs (NSAIDs), other opioids, or adjuvant analgesics (e.g., pregabalin, goshajinkigan, antidepressants, anticonvulsants, and antiarrhythmic agents) for cancer-related pain was allowed if the pain control was stable and the dosages were not expected to be changed during the study period. Exclusion criteria were peripheral neuropathies induced by other causes (e.g., spinal stenosis, diabetes mellitus, alcohol, and other drugs), unstable control of cancer-related pain, uncontrolled nausea/vomiting, and pregnancy/lactation. The study design complied with the Helsinki Declaration and all patients in this study provided written informed consent. This study was conducted with the approval of our institutional ethics committee and was registered with the University Hospital Medical Information Network Clinical Trials Registry (protocol ID UMIN000018147).

\section{Treatment plan}

This trial was a single-arm phase II study. Each TACT contained tramadol hydrochloride $37.5 \mathrm{mg}$ plus acetaminophen $325 \mathrm{mg}$. The total duration of protocol treatment with TACT was 28 days. The participants took one tablet twice daily, after breakfast and before sleep, for the first 7 days, with the dose increased to four tablets a day, taken after each meal and before sleep, for the subsequent 21 days. Continuation of TACT after the protocol treatment period of 28 days was permitted if the patient felt that it was effective against CIPN and was willing to continue the treatment. As prophylaxis against nausea and vomiting, which is reported as the most common side effect of tramadol/acetaminophen, ${ }^{7}$ prochlorperazine maleate $5 \mathrm{mg}$ was administered three times a day, after each meal, for the first 14 days.

\section{Endpoints}

Primary endpoint was the change in a numerical rating scale of neuropathic pain (NRSN) according to CIPN from baseline (day 0 ) to day 28 after the start of protocol treatment. The NRSN was reported by patients on the basis of the average severity of the neuropathic pain during the previous three days: 0 point representing no neuropathic pain and 10 point representing neuropathic pain of the worst imaginable severity.

Secondary endpoints were the clinical grade of peripheral sensory neuropathy and the safety of TACT during the treatment period. The clinical grade of peripheral sensory neuropathy and adverse events (AEs) of TACT were recorded in accordance with CTCAE 
v4.0. The peripheral sensory neuropathy was graded from 0 to 5 (grade 0, no symptoms; grade 1, asymptomatic with loss of deep tendon reflexes or paresthesia; grade 2, moderate symptom severity limiting instrumental activities of daily living (ADL); grade 3, severe symptom severity limiting self-care ADL; grade 4, life-threatening consequences; grade 5, death) in the worst severity during the period from last visit.

NRSN and clinical grade of the peripheral sensory neuropathy were determined at baseline and on days 7, 14, and 28. Any AEs were recorded at each scheduled visit and on any unscheduled visits.

\section{Examination of CYP2D6 SNPs}

As exploratory endpoints, the SNPs of CYP2D6 were assessed to evaluate their correlations with the efficacy and toxicity of TACT. Participants who provided consent to undergo this biomarker research were included in the analysis of the SNPs. The SNPs were measured using DNA extracted from white blood cells in a 3$\mathrm{mL}$ sample of whole blood. Exploration for CYP2D6 variations was performed using xTAG CYP2D6 Kit v3 RUO (Luminex Molecular Diagnostics, Canada), which is valid for checking the SNPs of CYP2D6 allele name ${ }^{\star} 1$ to ${ }^{\star} 12,{ }^{\star} 14,{ }^{\star} 15,{ }^{\star} 17,{ }^{\star} 29,{ }^{\star} 35,{ }^{\star} 41$, and duplication. The many genetic variants of CYP2D6 have been classified into the following four phenotypes by the combination of each allele: poor metabolizers (PMs), intermediate metabolizers (IMs), extensive metabolizers (EMs), and ultrarapid metabolizers (UMs).

\section{Statistical analysis}

The primary endpoint was defined as the mean change in NRSN from baseline to day 28. In the previous study of duloxetine, ${ }^{5}$ the mean neuropathic pain score (standard deviation $[\mathrm{SD}]$ ) at baseline in the duloxetine and control groups was 5.6 (1.6) and 6.1 (1.7), respectively, and the mean decrease in score from baseline to six weeks in the duloxetine group was 1.06. We hypothesized similar results, namely that the mean NRSN before treatment would be 6.0 (1.7) and the mean decrease in score would be 1.0. We needed a target sample size of 23 participants to complete this protocol treatment in this study to obtain $80 \%$ power and a 2 sided $\alpha$ of $5 \%$ to detect the target change using the paired sample $t$-test. Assuming a dropout rate of $20 \%$, the sample size in our study was set at 30 patients.

The secondary endpoints and subgroup analyses were analyzed using the dependent $t$-test or Wilcoxon's signed-rank test according to the statistical distribution for continuous variables, and Fisher's exact test for categorical variables. All statistical analyses were performed with SAS software version 9.4.

\section{Results}

Patient characteristics and treatment

We enrolled a total of 34 patients for this study between September 2014 and December 2015. Patient characteristics at baseline are summarized in Table 1 . Two patients (6\%) were concomitantly receiving adjuvant analgesic agents for CIPN, including pregabalin, goshajinkigan, and duloxetine, and four patients (12\%) were concomitantly receiving controlled-release oxycodone for cancer-related pain. None of the patients was receiving both the adjuvant analgesics and oxycodone.

\section{Efficacy}

All 34 participants received the intervention and were evaluated for efficacy and safety. The mean NRSN (SD) decreased from 5.53 (1.88) at baseline to 5.00 (2.37) on day 28 (Fig. 1). The mean decrement was 0.53 , which was not statistically significant ( $95 \%$ confidence interval -0.21 to $1.43 ; p=0.139$ ). However, 13 of the 23 patients (57\%), who completed the experimental treatment, showed an improvement in neuropathic pain score by at least 1 point (Fig. 2). There was no significant difference in efficacy between patients who were currently receiving the neurotoxic chemotherapies and those who had previously received them.

Table 1. Patient Baseline Characteristics $(n=34)$

\begin{tabular}{lc}
\hline Age (range), years & $67(46-74)$ \\
Gender: male/female & $22 / 12$ \\
Primary disease, $n(\%)$ & $19(55.9)$ \\
Colorectal & $12(35.3)$ \\
Pancreas & $3(8.8)$ \\
Stomach & \\
Causative agent, $n(\%)$ & $20(58.8)$ \\
$\quad$ Oxaliplatin & $10(29.4)$ \\
$\quad$ Nab-paclitaxel & $2(5.9)$ \\
Oxaliplatin and nab-paclitaxel & $2(5.9)$ \\
Paclitaxel & \\
Concomitant drugs for cancer-related pain, $n(\%)$ & $5(14.7)$ \\
$\quad$ NSAIDs & $4(11.8)$ \\
Other opioids & $2(5.9)$ \\
Adjuvant analgesics & \\
Neurotoxic chemotherapy, $n$ (\%) & $25(73.5)$ \\
$\quad$ Concurrent use & $9(26.5)$ \\
Previous use & \\
Numerical rating scale of neuropathic pain & \\
$\quad$ Mean \pm SD (range) & \\
Peripheral sensory neuropathy (CTCAE v4.0) & \\
$\quad$ Grade 2/3/4 & $19 / 15 / 0$ \\
\hline
\end{tabular}

CTCAE, common terminology criteria for adverse events; NSAIDs, nonsteroidal anti-inflammatory drugs; SD, standard deviation. 


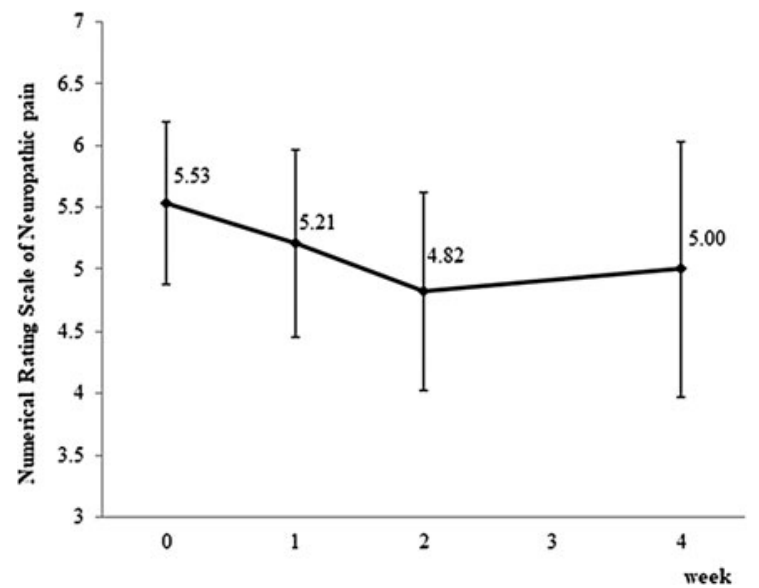

FIG. 1. Change in mean NRSN. NRSN during the treatment period tended to be lower than the score at baseline. The mean change from baseline to day 28 was $0.53(95 \% \mathrm{Cl}-0.21$ to 1.43; $p=0.139$; by paired $t$-test). Therefore, the primary endpoint was not met in this study. Error bars represent $95 \% \mathrm{Cls}$. $\mathrm{Cl}$, confidence interval; NRSN, numerical rating scale of neuropathic pain.

Other analyses in subgroups classified according to the primary location of the cancer, the causative neurotoxic agents (L-OHP or taxanes), and the concomitant use of other opioids or adjuvant analgesics revealed no statistically significant results.

\section{Adverse events}

Eight events in seven patients (21\%) were reported, namely nausea in three $(8.8 \%)$, somnolence in three $(8.8 \%)$, and malaise in two (5.9\%). One patient had both somnolence plus malaise. In each $\mathrm{AE}$, the clinical grade was 1 or 2 according to CTCAE v4.0, and there were no cases of any severe AE. However, all seven patients who encountered AEs discontinued the treatment. The median time of onset of AEs was day 7 (2-26) of treatment, and five patients discontinued the protocol treatment within seven days.

\section{Genetic polymorphism of CYP2D6}

Consent for biomarker research was obtained from 25 participants, and SNPs were examined. In this biomarker analysis cohort, 17 patients completed the study treatment. The mean NRSN (SD) in the cohort was $5.76(1.52)$ at baseline and $5.00(2.24)$ on day 28.

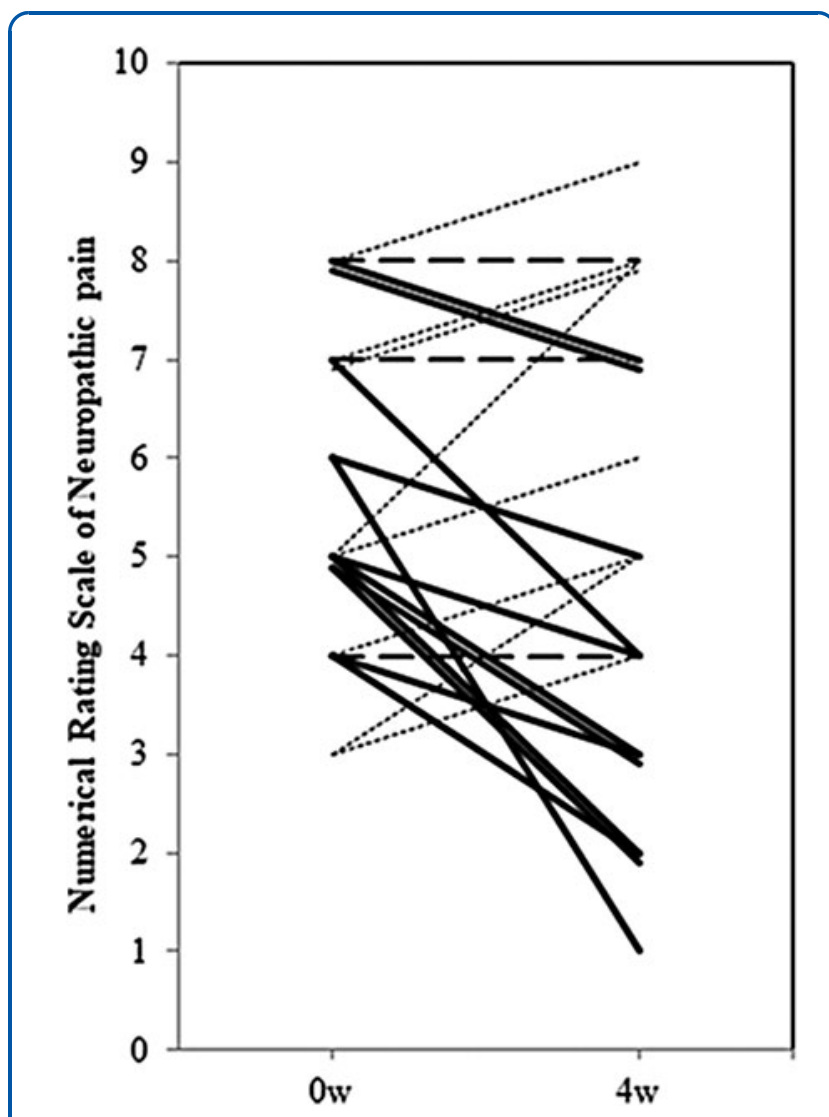

FIG. 2. NRSN at baseline and after treatment. Thirteen of 23 patients who completed the protocol treatment showed an improvement in NRSN by $\geq 1$ point from baseline to the end of the 28-day treatment period (solid lines). In three patients, the neuropathic pain score showed no change (broken lines). In seven patients, the neuropathic pain score increased compared with the baseline score (dotted lines).

Frequencies of the CYP2D6 SNPs are given in Table 2, and the distribution of the genotype and phenotype (enzyme activity) is summarized in Table 3. The allele frequencies were similar to those in a previous Japanese report. ${ }^{9}$ No UMs or PMs were identified

Table 2. Frequencies of CYP2D6 SNPs in This Study

\begin{tabular}{llc}
\hline & \multicolumn{1}{c}{ Enzyme function } & Frequency (\%) \\
\hline CYP2D6*1 & Normal & 30 \\
CYP2D6 2 & Normal & 22 \\
CYP2D6 5 & None (CYP2D6 deleted) & 8 \\
CYP2D6*10 & Reduced function & 36 \\
CYP2D6*41 & Reduced function & 4 \\
\hline
\end{tabular}

Duplication of CYP2D6*1 or *2 was not observed. 
Table 3. Effect of CYP2D6 Polymorphisms on Neuropathic Pain and Toxicity

\begin{tabular}{|c|c|c|c|c|c|c|}
\hline $\begin{array}{l}\text { Genotype } \\
(n=25)\end{array}$ & $\begin{array}{l}\text { Enzyme } \\
\text { activity }\end{array}$ & $n(\%)$ & $\begin{array}{c}\text { Completion } \\
\text { of treatment (\%) }\end{array}$ & $\begin{array}{l}\text { Mean change } \\
\text { in NRSN }\end{array}$ & $\begin{array}{c}\text { Responders }{ }^{a} \\
(\%)\end{array}$ & $\begin{array}{l}\text { Discontinued because } \\
\text { of toxicity (\%) }\end{array}$ \\
\hline $\begin{array}{l}* 1 /{ }^{*} 1 \\
{ }^{*} 1 /{ }^{*} 2 \\
* 2 / * 2\end{array}$ & EM & $7(28)$ & $6(86)$ & -0.67 & $8(53)$ & $3(16)$ \\
\hline $\begin{array}{l}* 1 / * 5 \\
* 1 / * 10 \\
* 2 / * 10 \\
*^{*} 2 /{ }^{*} 41\end{array}$ & & $12(48)$ & $9(75)$ & & & \\
\hline $\begin{array}{l}* 5 / * 10 \\
* 10 /{ }^{*} 10 \\
{ }^{*} 10 / * 41\end{array}$ & IM & $6(24)$ & $2(33)$ & -1.50 & $2(100)$ & $2(33)$ \\
\hline
\end{tabular}

a Patients who showed an improvement in NRSN by at least 1 point.

EM, extensive metabolizer; IM, intermediate metabolizer; NRSN, numerical rating scale of neuropathic pain.

in this study, because there were no patients with an active allele (duplication of ${ }^{\star} 1,{ }^{\star} 2$ ) or homozygous nonfunctioning alleles (e.g., ${ }^{*} 5 /{ }^{*}$ ). Seven $(28 \%)$ of the 25 patients had two active alleles $\left({ }^{\star} 1 /{ }^{\star} 1,{ }^{\star} 1{ }^{\star} 2\right.$, $\left.\star 2 /{ }^{\star} 2\right)$, and 12 patients $(48 \%)$ had just one active allele $\left({ }^{\star} 1 /{ }^{\star} 5,{ }^{\star} 1 /{ }^{\star} 10,{ }^{\star} 2 /{ }^{\star} 10,{ }^{\star} 2 /{ }^{\star} 41\right)$ that resulted in the EM phenotype. Six patients $(24 \%)$ had two alleles with a reduction in enzyme function $\left({ }^{\star} 5 /{ }^{\star} 10,{ }^{\star} 10 /{ }^{\star} 10,{ }^{\star} 10 /{ }^{\star} 41\right)$, which resulted in the IM phenotype. Among these six IM patients, two patients completed the study treatment, both of whom showed improved scores on neuropathic pain $(-1.0$ and -2.0 , respectively); two patients discontinued the protocol treatment due to the toxicity of TACT; and the remaining two patients discontinued the treatment due to transfer to other hospitals. Our findings suggested that tramadol/acetaminophen may be more effective in patients with the IM phenotype although at the cost of increased toxicity.

\section{Discussion}

CIPN is one of the most distressing adverse effects associated with cancer chemotherapy. The American Society of Clinical Oncology (ASCO) guidelines for CIPN recommend only duloxetine (one of SNRIs) in the treatment setting (moderately strong evidence). ${ }^{11}$ One retrospective study suggested that oxycodone (opioid analgesic) might be effective against CIPN. ${ }^{12}$ Tramadol has a dual mechanism of action (opioid and SNRI action). Therefore, we hypothesized that TACT, which included acetaminophen in addition, might be effective, and conducted this phase II study.

Although the primary endpoint of improvement in mean NRSN was not met in this study, some clinical benefit was nevertheless obtained in more than half of the patients. The NRSN improved in 13 (57\%) of the 23 patients who completed the protocol treatment. As a reference data, the duloxetine study reported that neuropathic pain decreased in $59 \%$ of the patients treated with duloxetine and $38 \%$ of the patients treated with placebo. ${ }^{5} \mathrm{Six}$ of these patients were willing to continue the treatment after the study period due to its efficacy, and two patients resumed intake of TACT later because they felt that the neuropathic pain had gotten worse after terminating the protocol treatment on completion of the clinical trial period. Changes of CIPN were not evaluated by the CTCAE in this study.

SNPs of CYP2D6, which is recognized as biomarkers for the metabolism of tramadol, were measured in 25 patients (74\%). CYP2D6 metabolizes tramadol to M1, which has 175-fold higher affinity for the $\mu$-opioid receptor than tramadol. ${ }^{13}$ This finding suggests that the analgesic effect of tramadol is lower in PM cases due to poor enzyme activity, whereas both its pain-relief effect and opioid-related toxicity are higher in UM than in the other metabolizer phenotypes. ${ }^{14}$ To our knowledge, the findings of tramadol for CIPN associated with CYP2D6 variations have not been reported. Although we saw neither PM nor UM cases in this study, 6 patients were categorized into the IM group (reduced metabolic function) and 19 patients were categorized into the EM group (normal metabolic activity). Because only two patients in the IM group completed this protocol treatment, this study could not show a significant difference in improvement of neuropathic pain scores between the IM and EM groups. However, there was a tendency for tramadol to show more efficacy in the IM patients than in the EM patients. It has been reported that tramadol compound has a stronger serotonin-reuptake inhibitor 
effect than M1. ${ }^{4}$ Because this SNRI effect impacts its efficacy against CIPN, the potential of CYP2D6 polymorphism as a biomarker requires further study.

Although AEs were observed in seven patients (21\%), no case had grade 3 or higher severity. All these patients with AEs discontinued the protocol treatment. It was assumed that all AEs in this study had been caused by tramadol, not by acetaminophen. Nausea is one of the most frequently encountered toxicities of tramadol, although in this study the incidence (8.8\%) was lower than expected. Concurrent use of a $5-\mathrm{HT}_{3}$ receptor blocker, which is frequently used as a prophylactic antiemetic in patients receiving cancer chemotherapy, might be effective in reducing tramadol-induced nausea, as the nausea is caused by the increase in the serotonin levels in synaptic clefts resulting from the SNRI action of tramadol. In this study, if a patient discontinued taking tramadol/ acetaminophen once because of an $\mathrm{AE}(\mathrm{s})$, he/she could restart the protocol treatment after the $\mathrm{AE}(\mathrm{s})$ had improved. However, none of the patients made an attempt to resume treatment, and the discontinuation rate of the study treatment was higher than expected.

This study had several limitations. First, the number of evaluable patients who completed the protocol treatment was small. Some patients experienced benefit from TACT, although promising factors could not be identified in the subgroup analyses because the number of patients in each subgroup was too small. Second, this study was not a randomized or blinded trial.

\section{Conclusions}

Although this study did not meet its primary endpoint, the severity of neuropathic pain was reduced in more than half of the patients. In current practice, TACT could be considered as a valid treatment option in patients with CIPN in whom duloxetine proves ineffective.

\section{Acknowledgments}

The authors gratefully thank the patients, their family members, and staff members who participated in this study for their support. Prof. Chikuma Hamada supported the statistical analysis of this study. The authors also thank Libby Cone, MD, MA, of DMC Japan for editing drafts of this article.

\section{Funding Information}

This study was supported by Janssen Pharmaceutical Kabushiki Kaisha Japan.

\section{Author Disclosure Statement}

The study was planned, carried out, and analyzed by the authors. No employees of Janssen Pharmaceutical K.K. were involved in these processes.

\section{References}

1. Ewertz M, Qvortrup C, Eckhoff L: Chemotherapy-induced peripheral neuropathy in patients treated with taxanes and platinum derivatives. Acta Oncol 2015:54:587-591.

2. Conroy $\mathrm{T}$, Desseigne $\mathrm{F}$, Ychou M, et al.: FOLFIRINOX versus gemcitabine for metastatic pancreatic cancer. N Engl J Med 2011;364: 1817-1825.

3. Von Hoff DD, Ervin T, Arena FP, et al.: Increased survival in pancreatic cancer with nab-paclitaxel plus gemcitabine. N Engl J Med 2013;369: 1691-1703.

4. Smith EM, Pang $\mathrm{H}$, Cirrincione $\mathrm{C}$, et al.: Effect of duloxetine on pain function, and quality of life among patients with chemotherapy-induced painful peripheral neuropathy: A randomized clinical trial. JAMA 2013; 309:1359-1367.

5. Raffa RB, Friderichs E, Reimann W, et al.: Opioid and nonopioid components independently contribute to the mechanism of action of tramadol, an 'atypical' opioid analgesic. J Pharmacol Exp Ther 1992; 260:275-285.

6. Mallet C, Daulhac L, Bonnefont J, et al.: Endocannabinoid and serotonergic systems are needed for acetaminophen-induced analgesia. Pain 2008;139:190-200.

7. Freeman R, Raskin P, Hewitt DJ, et al.: Randomized study of tramadol/acetaminophen versus placebo in painful diabetic peripheral neuropathy. Curr Med Res Opin 2007;23:147-161.

8. Liu YC, Wang WS: Human mu-opioid receptor gene A118G polymorphism predicts the efficacy of tramadol/acetaminophen combination tablets (Ultracet) in oxaliplatin-induced painful neuropathy. Cancer 2012;15: 1718-1725.

9. Nishida Y, Fukuda T, Yamamoto I, Azuma J: CYP2D6 genotypes in a Japanese population: Low frequencies of CYP2D6 gene duplication but high frequency of CYP2D6*10. Pharmacogenetics 2000;10:567-570.

10. Zahari Z, Ismail R: Influence of cytochrome P450, family 2, subfamily D, polypeptide 6 (CYP2D6) polymorphisms on pain sensitivity and clinical response to weak opioid analgesics. Drug Metab Pharmacokinet 2014;29: 29-43.

11. Hershman DL, Lacchetti C, Dworkin RH, et al.; American Society of Clinical Oncology: Prevention and management of chemotherapy-induced peripheral neuropathy in survivors of adult cancers: American Society of Clinical Oncology clinical practice guidelines. J Clin Oncol 2014;32:19411967.

12. Nagashima $M$, Ooshiro $M$, Moriyama $A$, et al.: Efficacy and tolerability of controlled-release oxycodone for oxaliplatin-induced peripheral neuropathy and the extension of FOLFOX therapy in advanced colorectal cancer patients. Support Care Cancer 2014;22:1579-1584.

13. Gillen C, Haurand M, Kobelt DJ, Wnendt S: Affinity, potency and efficacy of tramadol and its metabolites at the cloned human muopioid receptor. Naunyn Schmiedebergs Arch Pharmacol 2000;362: 116-121.

14. Zahari Z, Ismail R: Influence of cytochrome P450, family 2, subfamily D, polypeptide 6 (CYP2D6) polymorphisms on pain sensitivity and clinical response to weak opioid analgesics. Drug Metab Pharmacokinet 2014;29: 29-43.

Cite this article as: Naruge D, Nagashima F, Kawai K, Okano $\mathrm{N}$ Kobayashi T, Furuse J (2020) Tramadol/acetaminophen combination tablets in cancer patients with chemotherapy-induced peripheral neuropathy: A single-arm phase II study, Palliative Medicine Reports 1:1, 25-31, DOI: 10.1089/pmr.2020.0031. 
NSAID $=$ nonsteroidal anti-inflammatory drug

Abbreviations Used

$\mathrm{ADL}=$ activities of daily living

$\mathrm{AEs}=$ adverse events

CIPN = chemotherapy-induced peripheral neuropathy $\mathrm{Cl}=$ confidence interval

CTCAE $=$ Common Terminology Criteria for Adverse Events

CYP2D6 = cytochrome P450 2D6

$\mathrm{EM}=$ extensive metabolizer

$\mathrm{IM}=$ intermediate metabolizer

nab-PTX = nanoparticle albumin-bound paclitaxel

NRSN = numerical rating scale of neuropathic pain

$\mathrm{L}-\mathrm{OHP}=$ oxaliplatin

PTX = paclitaxel

$\mathrm{PM}=$ poor metabolizer

SNRI = serotonin-noradrenaline reuptake inhibitor

SNPs $=$ single nucleotide polymorphisms

$\mathrm{SD}=$ standard deviation

$\mathrm{TACT}=$ tramadol/acetaminophen combination tablet

$\mathrm{UM}=$ ultrarapid metabolizer

Publish in Palliative Medicine Reports

Palliative

Medicine

Reports

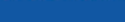

- Immediate, unrestricted online access

- Rigorous peer review

- Compliance with open access mandates

- Authors retain copyright

- Highly indexed

- Targeted email marketing

liebertpub.com/pmr 